\title{
ULTRASONIDO: UNA ALTERNATIVA EN LA EVALUACIÓN DEL DIAFRAGMA EN NIÑOS CON DISTROFIA MUSCULAR DE DUCHENNE
}

Dra. Lizbet Pérez $M^{(1)}$, Knlgo. Jorge Rodríguez $B^{(2)}$, Drs. Carlos Ubilla $P^{(3)}$, Claudia Díaz $S^{(4)}$, Rodrigo Arroyo $M^{(4)}$, María de los Ángeles Avaria $B^{(5)}$, Karen Kleinsteuber $\mathbf{S}^{(5)}$, Ester Ureta $\boldsymbol{H}^{(1)}$.

1. Radiólogo Pediatra, Hospital de Niños Roberto del Río. Santiago-Chile.

2. Kinesiólogo, Hospital de Niños Roberto del Río. Santiago-Chile.

3. Pediatra Broncopulmonar, Hospital de Niños Roberto del Río. Santiago-Chile.

4. Internos de Kinesiología, Escuela de Kinesiología. Facultad de Ciencias de la Salud, Universidad Católica del Maule. Chile.

5. Neuróloga Infantil, Hospital de Niños Roberto del Río. Santiago-Chile.

\section{ULTRASOUND, AN ALTERNATIVE IN THE EVALUATION OF THE DIAPHRAGM IN CHILDREN WITH DU- CHENNE MUSCULAR DYSTROPHY}

\begin{abstract}
The outcome of children with Duchenne muscular dystrophy (DMD) depends on respiratory involvement, so a timely assessment of the diaphragm is required. We propose ultrasound (US) imaging as an alternative in the evaluation of the diaphragm in children with DMD, correlating diaphragmatic thickness and excursion values yielded by the US study with pulmonary function tests. We conducted a case-control study including 27 children, 15 controls and 12 patients. Excursion and thickness of both hemidiaphragms were measured by U.S., and spirometry was performed. The DMD group showed less excursion and a significantly higher thickness of the right hemidiaphragm; $60 \%$ of patients showed spirometric restrictive pattern and FEV1, FVC, PEF and PIM values were significantly lower. We found a negative trend when correlating diaphragmatic excursion with pulmonary function tests. We conclude that the US technique is suitable for screening alterations in diaphragmatic excursion and thickness in children with $D M D$, since it provides supporting data to pulmonary function tests.

Keywords: Diaphragm, Diaphragmatic excursion, Duchenne muscular dystrophy, Thickness of the diaphragm, Ultrasound.
\end{abstract}

Resumen: El pronóstico de los niños con distrofia muscular de Duchenne (DMD) depende del compromiso respiratorio, siendo necesaria la evaluación oportuna del diafragma. Proponemos el ultrasonido (US) como alternativa en la evaluación del diafragma en niños con DMD, correlacionando los valores de excursión y grosor del diafragma obtenido con US, con las pruebas de función pulmonar. Realizamos un estudio de casos y controles, incluyendo 27 niños, 15 controles y 12 pacientes. Se midió excursión y grosor de ambos hemidiafragmas con US; se realizó espirometría y pimometría. El grupo DMD presentó menor excursión y grosor significativamente mayor del hemidiafragma derecho; el $60 \%$ mostró patrón restrictivo en la espirometría y los valores de VEF1, CVF, FEP y PIM fueron significativamente menores. Encontramos una tendencia negativa al correlacionar la excursión diafragmática con las pruebas de función pulmonar. Concluimos que el US es apto para pesquisar alteraciones en la excursión y grosor del diafragma en niños con DMD, apoyando las pruebas de función pulmonar.

Palabras clave: Diafragma, Distrofia Muscular Ducchenne, Excursión diafragmática, Grosor del diafragma, Ultrasonido.

Pérez L. Ultrasonido: una alternativa en la evaluación del diafragma en niños con distrofia muscular de Duchenne. Rev Chil Radiol 2011; 17(1): 37-43.

Correspondencia: Dra. Lizbet Pérez M. / lizbet.perez@gmail.com

Trabajo recibido el 12 de diciembre de 2010, aceptado para publicación el 18 de enero de 2011. 


\section{Introducción}

La distrofia muscular de Duchenne (DMD) es la enfermedad neuromuscular más frecuente de la infancia, afectando a 1/3.300 nacidos vivos de sexo masculino a nivel mundial y a 1/5.000 nacidos vivos en Chile ${ }^{(1)}$. Su pronóstico depende del grado de compromiso respiratorio(2), que se caracteriza por una progresiva caída de la presión inspiratoria máxima (PIM) y de la capacidad vital $(\mathrm{CV})^{(3)}$, asociándose a síndromes de hipoventilación (preferentemente nocturnos) que generan insuficiencia respiratoria y posteriormente causan la muerte en más del $70 \%$ de los pacientes ${ }^{(4)}$ lo que hace necesaria una evaluación oportuna del estado de la musculatura respiratoria.

Las radiografías de tórax son de poca utilidad para el diagnóstico temprano, pues si bien pueden mostrar la elevación del hemidiafragma afectado, este no es un signo específico debido al amplio rango de amplitud normal del diafragma. La fluoroscopía ha sido tradicionalmente utilizada para evaluar la movilidad del diafragma, sin embargo el hecho de ocupar radiación ionizante constituye una desventaja para el método, ya que son niños que necesitan repetidas evaluaciones ${ }^{(5)}$.

La polisomnografía es un examen adecuado para estudiar las condiciones fisiológicas del paciente en el sueño, sin embargo es de alto costo, requiere mucho tiempo y no es posible hacerla durante una consulta de rutina(2). La espirometría y la pimometría son pruebas validadas para evaluar el estado basal y el seguimiento de estos pacientes ${ }^{(6-9)}$.

El ultrasonido(US) aparece como una técnica simple y no invasiva que permite la caracterización morfológica del diafragma a través del modo $\mathrm{B}$ y la evaluación de sus movimientos mediante el modo M, por lo que ayudaría a diagnosticar sus disfunciones y se podría usar para seguimientos a largo plazo $(5,10,11)$.

\section{Objetivos}

Proponer el uso del US en la evaluación del diafragma en niños con DMD.

Correlacionar los valores de excursión y grosor del diafragma obtenidos a través del US, con las pruebas de función pulmonar en niños controles y con DMD.

\section{Material y Métodos}

Estudio de casos y controles, exploratorio, no experimental. Se estudiaron 27 niños, 12 niños con diagnóstico confirmado de DMD controlados en el Policlínico de Neurología del Hospital y 15 niños controles seleccionados del Colegio Arturo Merino Benítez de la comuna de Independencia en Santiago, con edades semejantes al grupo con DMD.

Se consideró como criterio de exclusión para el grupo control la existencia de antecedentes mórbidos, alteraciones músculo-esqueléticas severas o algún cuadro respiratorio, ya sea agudo o crónico. Para el grupo de casos se consideró que no estuvieran cursando con algún compromiso respiratorio o cardiológico intercurrente. Todos los padres o tutores firmaron un consentimiento informado para su participación en el estudio.

El estudio ultrasonográfico se realizó en el Servicio de Imaginología del Hospital Roberto del Río. Todos los exámenes de US fueron hechos por una radióloga pediatra staff de 5 años de experiencia. El equipo utilizado fue un Philips HD11 XE Ultrasound Machine, transductor curvo 2-5 MHz. En los 27 niños se realizaron mediciones de excursión y grosor de ambos hemidiafragmas a través de US, modo $\mathrm{M}$ y $\mathrm{B}$ respectivamente.

Con el paciente en decúbito supino se realizó la evaluación de ambos hemidiafragmas, mediante un abordaje subxifoídeo con modo B para evaluación general de los hemidiafragmas.

Con el paciente en decúbito supino, respirando a volumen corriente, se realizó un abordaje intercostal para la evaluación de ambos hemidiafragmas por separado y con el modo $\mathrm{M}$ se midió la excursión diafragmática (Figura 1). Con el Modo B se midió el espesor del tercio posterolateral del hemidiafragma, en volumen corriente y en inspiración máxima.

Los valores de peso fueron obtenidos con ropa y la altura fue medida sin zapatos. En los niños con DMD que estaban incapacitados para ponerse de pies, se consideraron los valores indicados en la ficha clínica durante su último control.

La evaluación espirométrica se realizó con un espirómetro portátil Microlab modelo 3.300, de acuerdo a los criterios de ATS y sus valores teóricos fueron calculados según Gutiérrez ${ }^{(12)}$. La pimometría se tomó con un manómetro anaeroide Smith $\left(0-120 \mathrm{~cm} \mathrm{H}_{2} \mathrm{O}\right)$, según el método propuesto por Black y Hyatt, usando en cálculo de valores teóricos según Wilson ${ }^{(8)}$. Los valores de VEF1, CVF, FEP y PIM pesquisados fueron recopilados en una ficha.

Se realizó un análisis estadístico con el software SPSS para Windows v.17.0 y Medcalc v.11.0. Se utilizaron mediciones paramétricas o no paramétricas dependiendo de la distribución de las variables. Las variables fueron expresadas como promedios (DE) o medianas (rango) y la asociación de las variables fue evaluada usando el coeficiente de correlación de Pearson.

\section{Resultados}

Se estudió un total de 27 niños (15 controles y 12 pacientes con DMD), cuyas características se muestran en la Tabla I. Se efectuaron mediciones de US a todos los niños, excluyendo en el análisis de la excursión diafragmática a un niño con DMD, ya que no se logró visualizar con claridad el diafragma debido a su contextura. Por otra parte, no fue posible 


\section{Ultrasonografía Modo M}

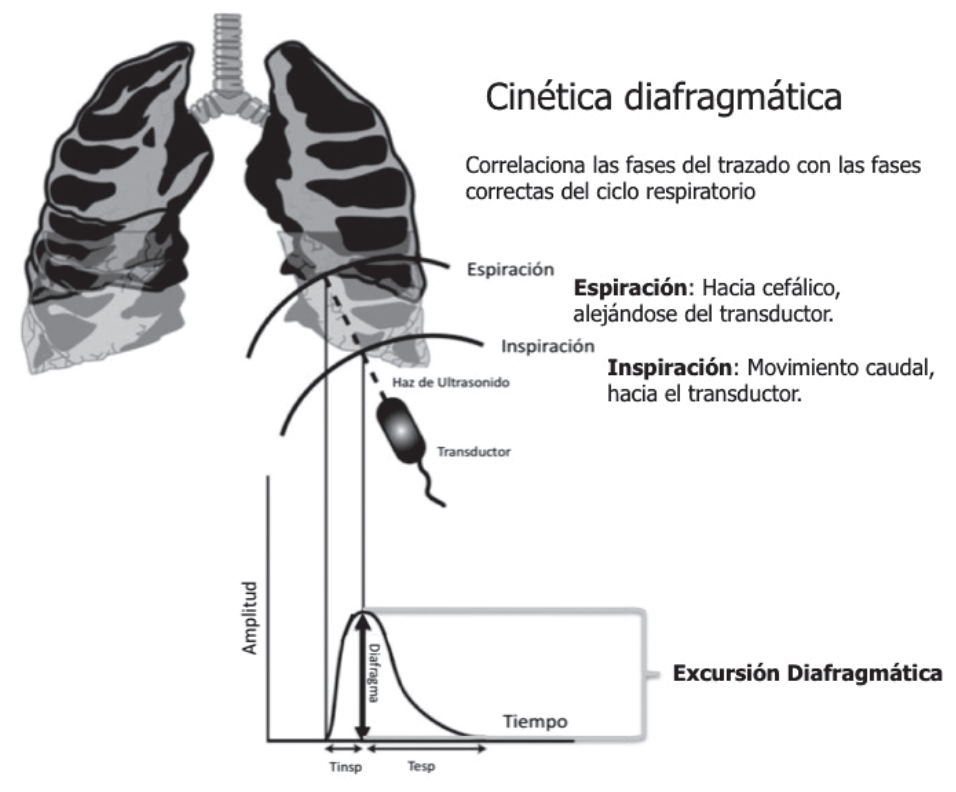

Figura 1. Esquema de cinética diafragmática y US modo M. Adaptado de Epelman M Pediatr Radiol. 2005; 35: 661-667.

Tabla I. Características generales de la muestra.

\section{CARÁCTERÍSTICAS GENERALES DE LA MUESTRA}

\begin{tabular}{llll|}
\hline CARÁCTERÍSTICA & $\begin{array}{l}\text { GRUPO CONTROL } \\
\mathbf{n = 1 5}\end{array}$ & $\begin{array}{l}\text { GRUPO DMD } \\
\mathbf{n = 1 2}\end{array}$ & $\mathbf{p}$ \\
\hline Edad (años) & $10,7( \pm 4)$ & $11( \pm 4)$ & $\mathrm{n} / \mathrm{s}$ \\
\hline Peso $(\mathrm{kg})$ & $44,2( \pm 15)$ & $37,8( \pm 13)$ & $\mathrm{n} / \mathrm{s}$ \\
\hline Altura $(\mathrm{cm})$ & $150,4( \pm 21)$ & $129,9( \pm 19)$ & $\leq 0,05$ \\
\hline IMC $\left(\mathrm{kg} / \mathrm{m}^{2}\right)$ & $19,0( \pm 3)$ & $21,4( \pm 3)$ & $\leq 0,05$ \\
\hline
\end{tabular}

Los resultados están expresados como promedios con su desviación estándar.

realizar pruebas de función pulmonar a 2 niños con DMD, por lo que se excluyeron de los análisis de espirometría y PIM.

En general la excursión del grupo con DMD fue menor que en el grupo control para ambos hemidiafragmas, como se muestra en la Gráfico 1 y se ejemplifica en la Figura 2.

Al describir ambos hemidiafragmas, el grupo control presentó mayor excursión del hemidiafrag- ma derecho, con una mediana de $1,6 \mathrm{cms}$ (rango 1,1 a 2,5), el con DMD mostró mayor excursión del hemidiafragma izquierdo, con una mediana de 1,3 cm (rango 0,9 a 1,6).

Al dividir la muestra en 2 grupos (mayores y menores de 10 años) y comparar las excursiones de ambos hemidiafragmas, encontramos que el hemidiafragma derecho presenta menor excursión en niños con DMD mayores de 10 años, con una diferencia 
promedio de 0,8+/-0,7 cms ( $p<0,05)$ (Gráfico 2).

El grosor del hemidiafragma derecho fue significativamente mayor a volumen corriente e inspiración máxima en el grupo DMD con un valor de $0,5 \mathrm{~cm}$ (rango $0,3-0,9)(p=0,001)$. En los niños con DMD mayores de 10 años este comportamiento es aún más notorio, con una diferencia promedio de 0,3 $\mathrm{cm}(\mathrm{p}=0,001)$ (Gráfico 2), como se ejemplifica en la Figura 3.

La espirometría mostró patrones restrictivos en el $60 \%$ de los niños con DMD, a su vez los valores de la espirometría fueron significativamente menores que en los niños controles: VEF1 (mediana de 2,5 L control vs 1,4 L DMD), CVF (mediana de 2,6 L control vs 1,6 L DMD) y FEP (mediana de $308 \mathrm{~L} / \mathrm{s}$ control vs $185 \mathrm{~L} / \mathrm{s} \mathrm{DMD})(\mathrm{p}=0,002)$. La PIM presentó una mediana $67,5 \mathrm{~cm}$ de $\mathrm{H}_{2} \mathrm{O}$ (rango 49-105), significativamente menor en niñ̃os con DMD $(p=0,008)$ (Gráfico 3).
Existe una correlación de 0,5 entre la excursión del hemidiafragma derecho con el VEF1, CVF y PIM en el grupo control, no significativas $(p=0,06$; $p=0,07 ; p=0,08$ ) (Gráfico 4). A su vez, la excursión del hemidiafragma derecho tuvo una correlación con la edad de 0,48 no significativa $(p=0,07)$ en el grupo control.

Al correlacionar el grosor del hemidiafragma con las pruebas de función pulmonar, no fue significativa para ninguno de los valores tanto en niños controles como en niños con DMD.

Al comparar excursión y grosor del hemidiafragma derecho encontramos una correlación negativa en inspiración máxima, no significativa, de $0,7(p=0,08)$ en niños con DMD mayores de 10 años (Gráfico 5).

\section{Discusión}

En la presente investigación demostramos que la US modo M permite una óptima evaluación de la
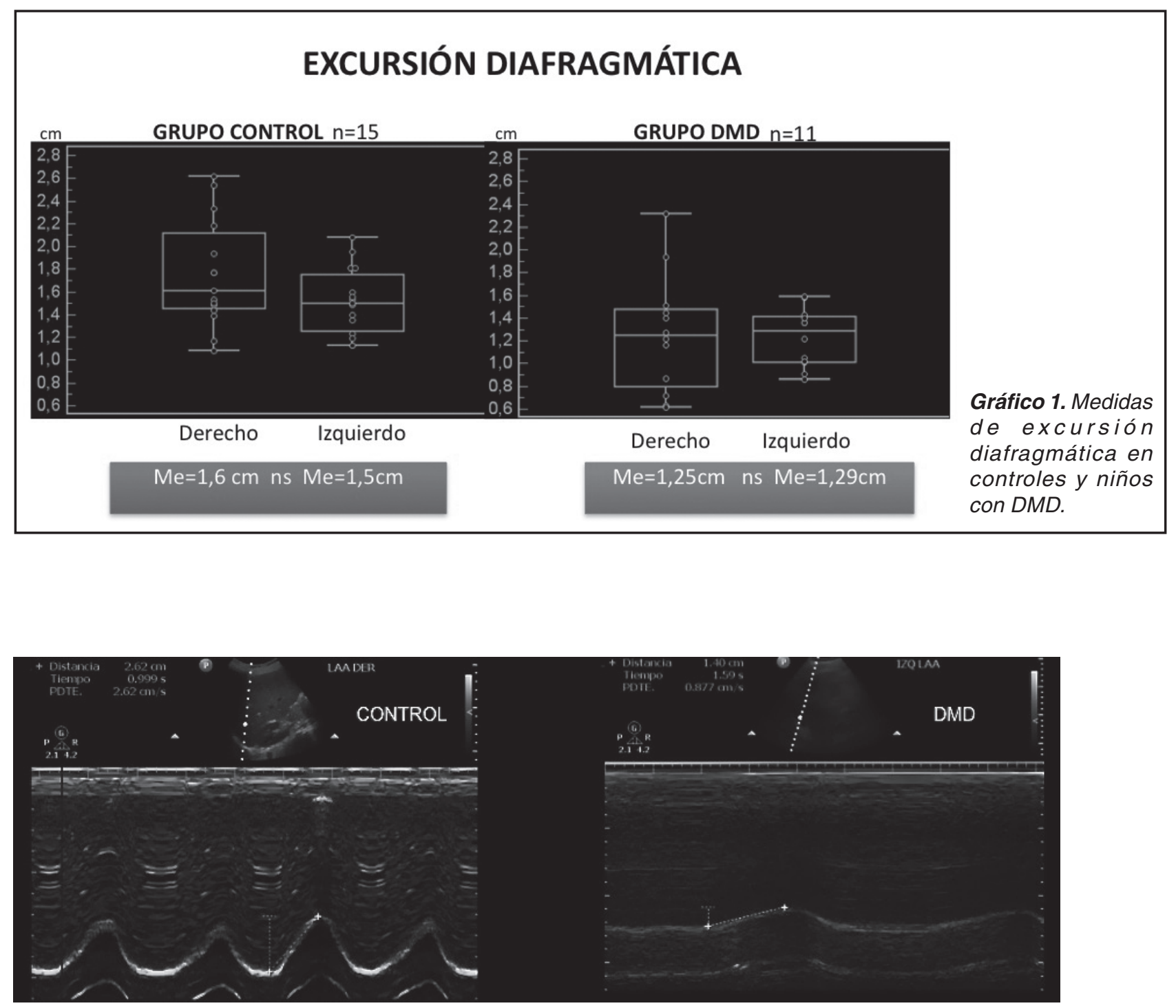

Figura 2. Medición con modo M de la excursión diafragmática en un control y en paciente con DMD, se aprecia una clara disminución de la curva en el niño con DMD. 

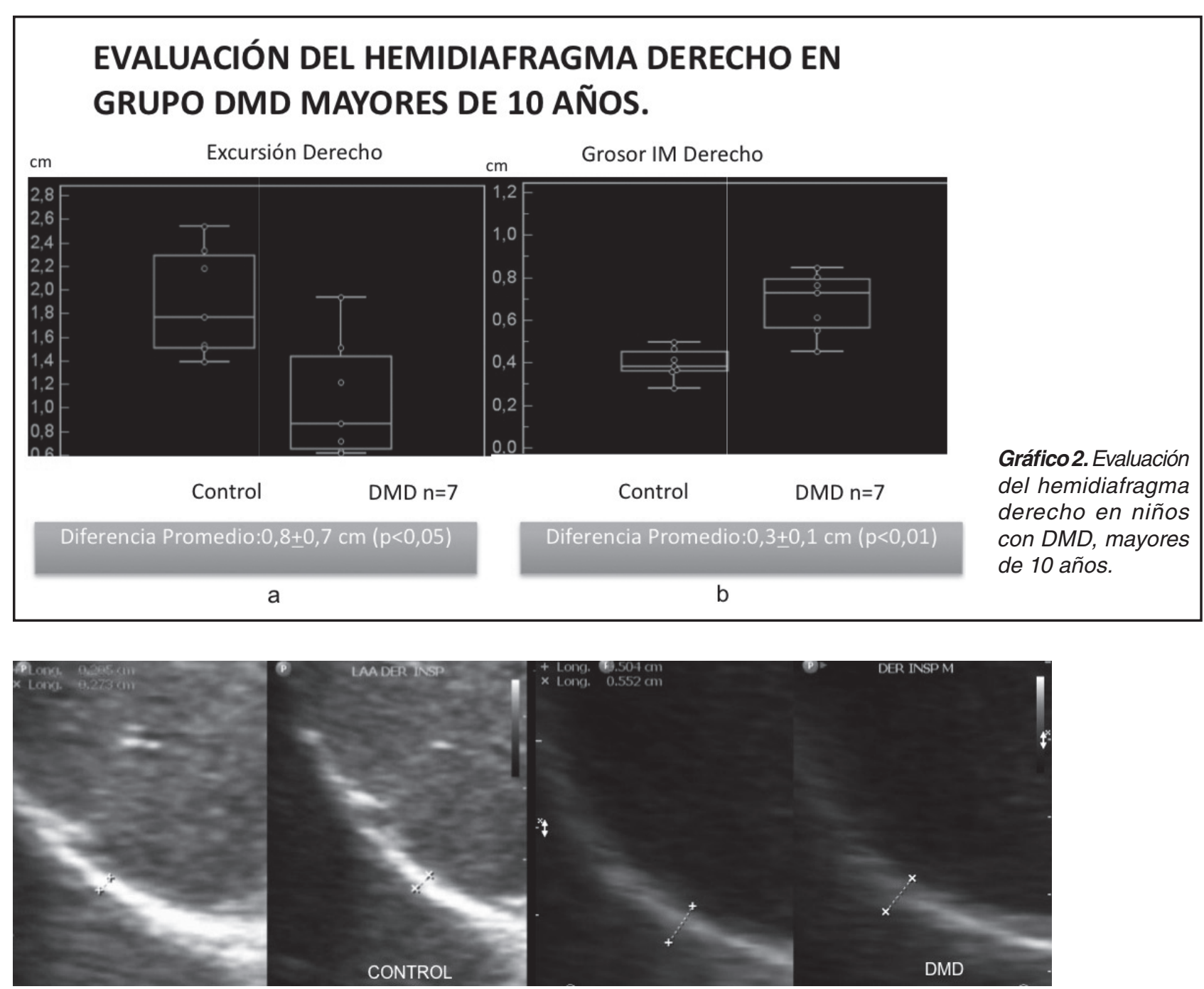

Figura 3. Medición con modo $\mathrm{M}$ del grosor del hemidiafragma derecho en inspiración máxima en control y paciente con DMD; se aprecia un aumento del espesor en el niño con DMD.

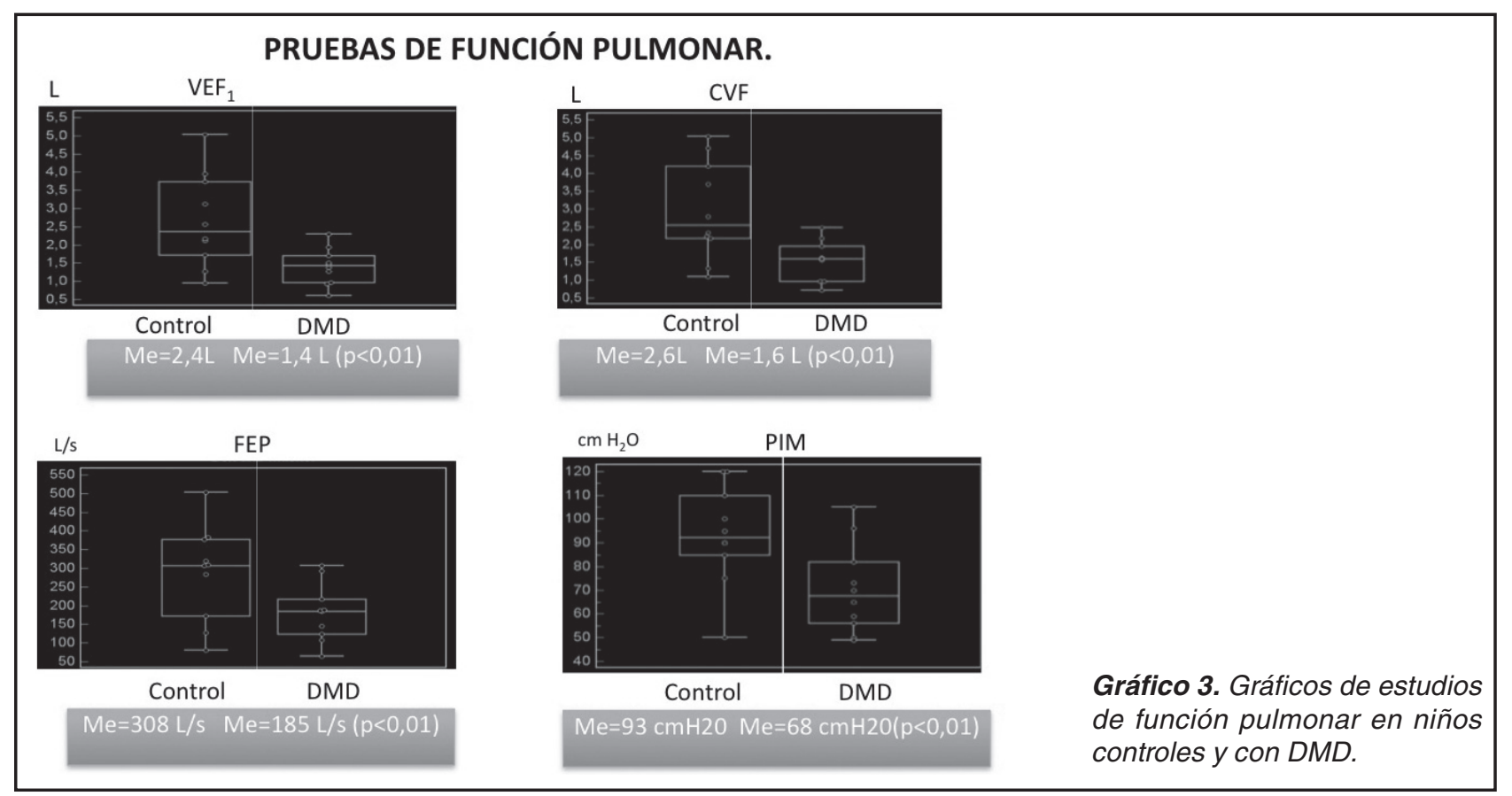




\section{CORRELACIÓN ENTRE LAS PRUEBAS DE FUNCIÓN PULMONAR Y EXCURSIÓN DIFRAGMÁTICA. CONTROLES.}

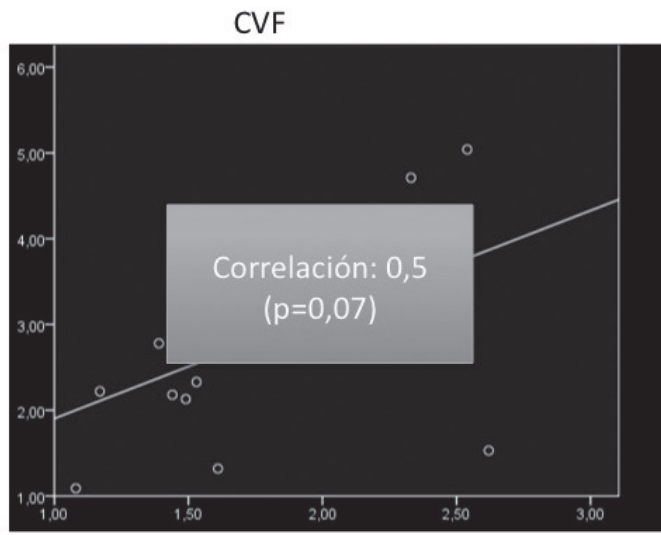

Excursión Derecha

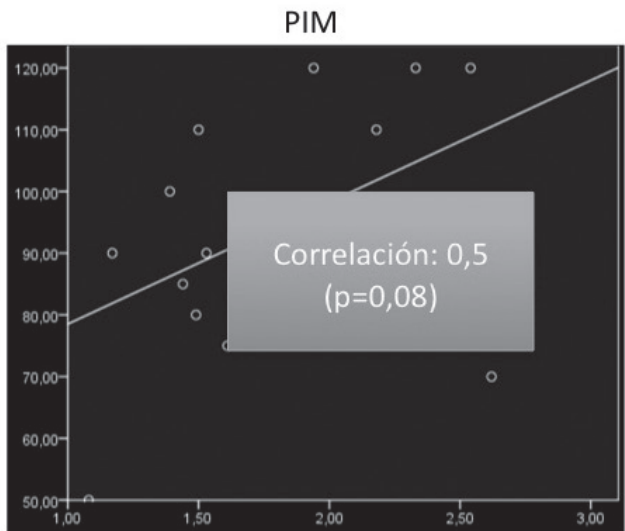

Excursión Derecha
Gráfico 4. Correlación las pruebas de función pulmonar $y$ excursión diafragmática en niños controles.

\section{CORRELACIÓN ENTRE LA EXCURSIÓN DIAFRAGMÁTICA Y \\ EL GROSOR DEL HEMIDIAFRAGMA DERECHO EN \\ MAYORES DE 10 AÑOS. DMD.}

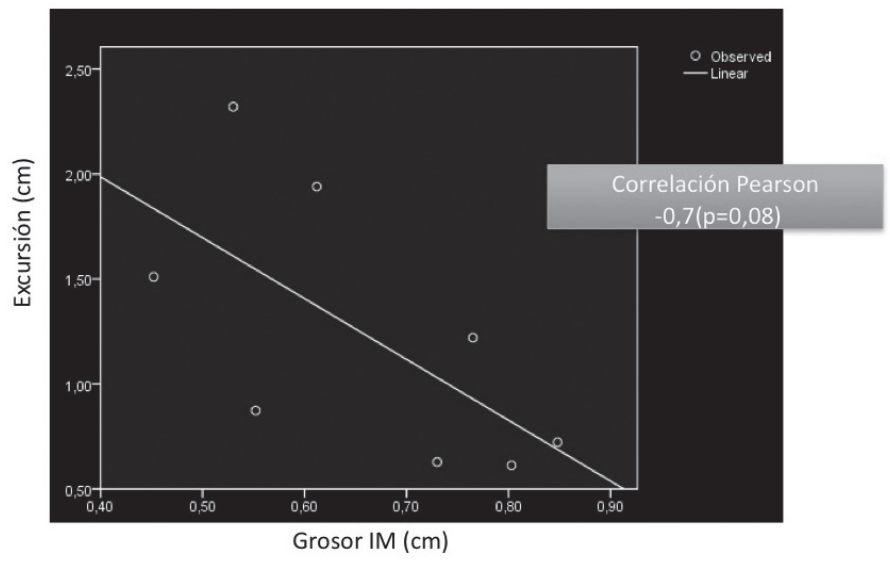

Gráfico 5. Correlación entre la excursión diafragmática y el grosor del hemidiafragma derecho en niños con DMD, mayores de 10 años.

excursión del diafragma en niños con DMD, la cual es menor en ambos hemidiafragmas al ser comparados con niños normales de la misma edad, lo que es más evidente en el hemidiafragma derecho de los niños con DMD mayores de 10 años, por tanto en los niños con DMD el hemidiafragma más móvil es el izquierdo, al contrario de los niños controles. En un estudio realizado por Cohen y cols refieren que un $65 \%$ de los sujetos sanos presentan mayor movilidad del hemidiafragma derecho y en un $15 \%$ del izquierdo ${ }^{(10)}$. Creemos que este comportamiento en la movilidad en los niños con DMD puede producirse como respuesta compensatoria a la disminución de la excursión del hemidiafragma derecho en etapas tempranas de la enfermedad.

No encontramos correlación entre la excursión diafragmática con pruebas de función pulmonar en niños con DMD, mientras que en los niños controles sólo encontramos una tendencia. Boussuges y cols encontraron una correlación entre la excursión del hemidiafragma derecho, el VEF1 y la CVF en una muestra de 210 sujetos adultos sanos ${ }^{(11)}$. Asumimos que nosotros encontramos sólo una tendencia por el bajo número de individuos de la muestra y porque las mediciones de excursión no fueron realizadas bajo las mismas condiciones de esfuerzo, como la 
espirometría y la PIM. Además los niños con DMD presentan una alta variabilidad de función pulmonar entre ellos, posiblemente por los diferentes grados de deterioro de la musculatura respiratoria, lo que es parte de la historia natural de la DMD y ha sido demostrada en estudios anteriores ${ }^{(6,7)}$; por otro lado, un número importante de los niños con DMD reciben tratamiento kinésico domiciliario periódicamente, donde entrenan la musculatura inspiratoria, lo cual incide sobre los valores de función pulmonar.

En cuanto al grosor del diafragma es significativamente mayor en niños con DMD y esta diferencia es más evidente en los mayores de 10 años. El grosor del diafragma en niños controles se correlaciona significativamente con el IMC al estar corregido por edad, indicando la incidencia del peso en dicho grosor. En el grupo con DMD la incidencia del peso sobre el grosor fue lo que marcó una tendencia positiva, tomando en cuenta que de los 12 niños con DMD, 7 tenían sobrepeso y 3 eran obesos. El aumento de grosor del diafragma en niños con DMD probablemente es debido a la pseudohipertrofia, que se ha demostrado en otros grupos musculares ${ }^{(13)}$. Planteamos que la pseudohipertrofia muscular podría estar influenciada por el aumento de la masa corporal.

No existió una correlación negativa esperable entre el grosor del diafragma y las pruebas de función pulmonar en niños con DMD. La zona de medición del hemidiafragma en nuestro estudio correspondió al tercio posterolateral, creemos necesario un punto de medición más definido. Encontramos una tendencia negativa al correlacionar el grosor del hemidiafragma derecho en los niños con DMD con la excursión de éste, demostrando que a medida que aumenta el grosor disminuye la movilidad del hemidiafragma.

En los 2 niños con DMD a los que no fue posible realizarles mediciones de función pulmonar debido a la alteración cognitiva que limitaba su cooperación, las mediciones de excursión y grosor pudieron ser evaluadas en ellos mediante US, demostrando que ésta es capaz de ser realizada a cualquier niño con o sin alteración cognitiva. Rideau y cols refieren que ésta es una de las limitaciones de la espirometría en niños con $\mathrm{DMD}^{(6)}$.

\section{Conclusión}

La US, en sus modos B y M, demostró ser una herramienta apta para pesquisar alteraciones en la excursión y grosor del diafragma en niños con DMD sobre 10 años de edad, período en el cual se expresan con mayor claridad las alteraciones diafragmáticas. Puede servir como apoyo a mediciones de función respiratoria o ser una herramienta principal de evaluación, en los pacientes con limitaciones para realizar maniobras voluntarias requeridas para técnicas como espirometría o pimometría. Por tratarse de una técnica no invasiva y que no ocupa radiación ionizante, podría además ser utilizada como seguimiento de la evolución de la enfermedad.

\section{Bibliografía}

1. Cruz-Coke R y Moreno R. Genetic epidemiology of single gene defects in Chile. J. Med. Genet. 1994. 31: 702-706.

2. Toussaint M, Steens M and Philippe Soudon. Muscular Dystrophy Hypercapnia in Patiens with Duchcenne Lung Function Accurately Predicts. Chest 2007. 131: 368-375.

3. Beck $\mathrm{J}$ and Weinberg J. Diaphragmatic function in advanced Duchenne muscular dystrophy. Neuromuscular Disorders. 2006. 16: 161-167.

4. Simonds AK. Respiratory Complications of Muscular Dystrophies. 23 (3): 231-238, 2002.

5. Epelman M. and Navarro O. M-mode sonography of diaphragmatic motion: description of technique and experience in 278 pediatric patiens. Pediatr. Radiol. 2005. 35: 661-667.

6. Rideau $Y$ and Jankowski LW. Respiratory function in the muscular dystrophies. Muscle \& Nerve 1981. 4 (2): 155-164.

7. Phillips M. Changes in Spirometry Over Time as a Prognostic Marker in Patiens with Duchenne Muscular Dystrophy. Am. J. Respir. Crit. Care Med. 2001.164 (12): 2191-2194.

8. Wilson SH, Cooke NT, Edwards RH and Spiro SG. Predicted normal values for maximal respiratory pressures in caucasian adults and childrens. Thorax 1984. 39: 535-538.

9. Tersi N, Orlikowski D. Measuring inspiratory muscle strength in neuromuscular disease: one test or two? Eur Respir J. 2008. 31: 93-98.

10. Cohen E, Mier A, Heywood P, Murphy K, Boultbee $J$ and Guz A. Excursion-volume relation of the right hemidiaphragm measured by ultrasonography and respiratory airflow measurements. Thorax 1994. 49 (9): 885-889.

11. Boussuges A, Gole Y. Diaphragmatic motion studied by M-mode ultrasonography: methods, reproducibility and normal values. Chest 2009.135 (2): 391-400.

12. Gutierrez M, Rioseco F, Rojas A y Casanova D. Determinación de valores espirométricos en una población chilena normal mayor de 5 años, a nivel del mar. Rev. Med. Chile 1996. 124: 1295-1306.

13. PF De Bruin, J Ueki, A Bush, Y Khan, A Watson, NB Pride. Diaphragm thickness and inspiratory strength in patines with Duchenne Musculary Dystrophy. Thorax 52: 472-475, 1997.

Nota: Este estudio formó parte de la Tesis para optar al grado de Licenciado en Kinesiología. "Estudio Diafragmático Mediante Ultrasonografía Modo B y Modo M en Niños con Distrofia Muscular de Duchenne" de los alumnos Rodrigo Arroyo Mancilla y Claudia Díaz Sanhueza. Profesor Guía Klgo. Jorge Rodríguez Borges. Escuela de Kinesiología. Facultad de Ciencias de la Salud. Universidad Católica del Maule. 2009. 